$1986,35,2$

\title{
ON THE EVOLUTION OF SINUSOIDAL PULSES IN A NONLINEAR MEDIUM WITH FADING MEMORY
}

\author{
(Presented by N. Alumäe)
}

The propagation of the one-dimensional longitudinal deformation wave type pulses in the nonlinear viscoelastic (hereditary) medium with arbitrary fading memory, is considered. The input is formulated through the velocity, which is given as a sinusoidal function. The asymptotic solution is constructed as a sum of a stationary component and two transient components. The stationary component consists of a first harmonic and two second harmonics. One of the transient components describes a distortion behind the front, whereas the other one describes the formation of the «tail» of the pulse; both of them decay rather quickly due to the fading memory of the medium.

\section{Introduction}

The «semistationary» technique of ultrasonic nondestructive testing of materials has been successfully used by many authors for analysing the physical properties of several materials $\left[{ }^{1-3}\right]$. According to this technique, long sinusoidal pulses are generated and the evolution of the stationary («central») part of these pulses is investigated without paying any attention to the distortions at the beginning and at the end of each pulse caused by switching in and out of the generator of the pulses. In such experiments the nonlinearity of the medium is usually described with the aid of the method presenting the stationary part of the pulse as a sum of the basic (first) and higher-order harmonics.

On the other hand, the near-front asymptotic methods have been developed for the description of the evolution of the short pulses in nonlinear hereditary media with fading memory $\left[{ }^{4,5}\right]$. In $\left[{ }^{6,7}\right]$ the ray method has been used in order to obtain the approximate evolution equations for the description of the high-frequency and the low-frequency relaxation processes in the nonlinear standard viscoelastic solids.

In the above-mentioned papers $\left[{ }^{4-7}\right]$ the integro-differential equations having regular kernels have been used. In $\left[{ }^{8,9}\right]$ the numerical solutions have been obtained in case of singular kernels. It has been shown in $\left[{ }^{9}\right.$ ] that in case of the simplest singular model, the model with Abel kernel, the phase velocity of the low-frequency waves is very small, which might be interpreted as a defect of the model; however, in case of the singular kernel, proposed by Rabotnov, it is possible to remove this defect. The stationary solutions in case of the Green-Rivlin model of the materials with memory have been investigated in [ $\left.{ }^{10}\right]$ by making use of a perturbation method.

In $\left.{ }^{5}\right]$ the attention has been drawn to the fact that in case of the sinusoidal input, the forementioned two types of methods - the near- 
front asymptotic methods and the stationary technique - might bè connected by constructing the solution in the form of a sum of a stationary part and of the transient components. In this paper we shall present the second-order asymptotic formulae of realization of the scheme proposed in $\left.{ }^{5}\right]$ in case of the one-dimensional longitudinal deformation waves in the nonlinear hereditary medium, both in case of the regular and of the singular kernel. It is shown that the stationary component consists of a basic and two second harmonics. One of these second harmonics has the same phase velocity and the double damping decrement of the basic harmonic, but the other one propagates with a different phase velocity, having also a different damping decrement.

\section{The statement of the problem}

Let us denote by $t$ the time, by $X$ the Lagrangian coordinate and by $U(X, t)$ the longitudinal displacement. Let the dot denote a derivative with respect to $t$ and the prime a derivative with respect to $X$. We shall use the initial conditions

$$
U(X, 0)=0, \quad U \cdot(X, 0)=0
$$

and the constitutive equation

$$
Q\left(U^{\prime}\right)=E^{-1}\left[\sigma(X, t)+\int_{0}^{t} K(t-\tau) \sigma(X, \tau) d \tau\right],
$$

where $\sigma(X, t)$ denotes the longitudinal stress, $E=$ const $>0$ and $Q$ is a continuous function of $U^{\prime}(X, t)$. The constitutive equation together with the equation of motion

$$
\varrho_{0} U \cdot(X, t)=\sigma^{\prime}(X, t),
$$

gives us the following governing equation

$$
U \cdot(X, t)-c^{2} U^{\prime \prime}(X, t) q\left(U^{\prime}\right)+\int_{0}^{t} K(t-\tau) U^{*}(X, \tau) d \tau=0 .
$$

Here $q\left(U^{\prime}\right)=d Q\left(U^{\prime}\right) / d U^{\prime}$ and $c=\left(E / \varrho_{0}\right)^{1 / 2}$ denotes the wave front velocity (the instantaneous velocity $\left[{ }^{3,7}\right]$ ), $\varrho_{0}$ being the density. $K(t)$ is the kernel function, which we suppose to be a positive and continuous decreasing function satisfying the condition $\lim _{t \rightarrow \infty} K(t)=0$.

We shall consider a wave which propagates in the $X$-axis positive direction and is generated by the input

$$
U \cdot(0, t)=-\varepsilon c\left[H(t)-H\left(t-t_{0}\right)\right] \sin \Omega t, \quad|\varepsilon| \ll 1,
$$

where $H(t)$ denotes the Heaviside step-function, $\Omega t_{0}=n \pi$ and $n$ is an integer.

We shall construct the solution of the equation (1.4) in the form $\left.{ }^{5}\right]$

$$
\begin{aligned}
& U \cdot(X, t)=U_{S}^{\cdot}(X, t)+U_{N}(X, t), \\
& U_{S}(X, t)=\varepsilon c\left[H\left(t_{1}\right)-H\left(t_{1}-t_{0}\right)\right] F_{0}^{*}(X, t), \\
& U_{N}(X, t)=\varepsilon c\left[H\left(t_{1}\right) F_{1}(X, t)+H\left(t_{1}-t_{0}\right) F_{2}(X, t)\right],
\end{aligned}
$$

where $t_{1}=t-X / c, U_{S}^{\cdot}(X, t)$ and $U_{N}(X, t)$ denote, correspondingly, the stationary part and the sum of two transient components of the solution. 


\section{Stationary part of the solution}

We shall construct the function $F_{0}^{*}(X, t)$ for the region $t_{1} \geqslant 0$ following [5] as the solution of the equation

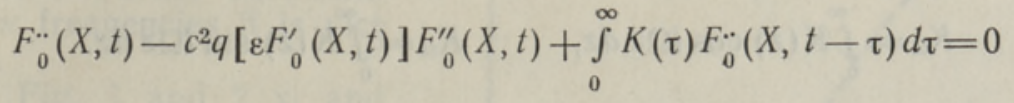

in the form of an expansion

$$
F_{0}^{\cdot}(X, t)=F_{01}(X, t)+\varepsilon F_{02}(X, t)+\ldots,
$$

which satisfies the condition

$$
F_{0}(0, t)=-\sin \Omega t .
$$

Here

$$
q\left[\varepsilon F_{0}^{\prime}(X, t)\right]=1+\varepsilon k_{1} F_{0}^{\prime}(X, t)+\ldots .
$$

The first-order term $F_{01}(X, t)$ is, in fact, the well-known solution [ ${ }^{1}$ ] of the linear equation obtained from (2.1) by taking into consideration only the first term of the expansion (2.4). It may be presented in the form

$$
\begin{gathered}
F_{01}(X, t)=-\exp \left[-a_{1}(\Omega) \Omega X / c\right] \sin \left[\Omega\left(t-X / c_{1}\right)\right], \\
c_{1}=c / b_{1},
\end{gathered}
$$

$$
\begin{array}{ll}
a_{1}(\Omega)=\left\{\frac{1}{2}[N(\Omega)-M(\Omega)]\right\}^{1 / 2}, & b_{1}(\Omega)=\left\{\frac{1}{2}[N(\Omega)+M(\Omega)]\right\}^{1 / 2}, \\
M(\Omega)=1+K_{c}(\Omega), & N(\Omega)=\left[M^{2}(\Omega)+K_{s}^{2}(\Omega)\right]^{1 / 2},
\end{array}
$$

$$
\begin{aligned}
& K_{c}(\Omega)=\int_{0}^{\infty} K(t) \cos \Omega t d t \\
& K_{s}(\Omega)=\int_{0}^{\infty} K(t) \sin \Omega t d t
\end{aligned}
$$

The first-order term $F_{01}(X, t)$ satisfies the boundary condition (2.3) and describes the propagation of a stationary sine wave in the linear hereditary medium with the phase velocity $c_{1}(\Omega)$. From the point of view of the nonlinear theory this sine wave is the basic or the first harmonic of the stationary part of the solution.

The second-order term $F_{02}(X, t)$ has the form

$F_{02}(X, t)=-\frac{1}{2} k_{1} \sum_{l=1}^{2} \exp \left(-\gamma_{l} X\right)\left(\alpha \cos 2 \Omega t_{l s}-\beta \sin 2 \Omega t_{l s}\right)(-1)^{l-1}$,

where

$$
\begin{array}{ll}
t_{l s}=t-X / c_{l} ; & c_{l}=c / b_{l}, \quad \gamma_{l}=2 a_{l} \Omega / c, \\
\alpha=\left(A_{1} S_{2}-A_{2} S_{1}\right) / P, & \beta=\left(A_{1} S_{1}+A_{2} S_{2}\right) / P, \\
P=S_{1}^{2}+S_{2}^{2}, & S_{2}=K_{2 c}-K_{c}, \\
S_{1}=K_{2 s}-K_{s}, & A_{2}=\frac{1}{2} a_{1}\left(3 b_{1}^{2}-a_{1}^{2}\right), \\
A_{1}=\frac{1}{2} b_{1}\left(3 a_{1}^{2}-b_{1}^{2}\right), &
\end{array}
$$




$$
\begin{array}{ll}
a_{l}=\left[\frac{1}{2}\left(N_{l}-M_{l}\right)\right]^{1 / 2}, & b_{l}=\left[\frac{1}{2}\left(N_{l}+M_{l}\right)\right]^{1 / 2}, \\
M_{l}=1+K_{l c}, & N_{l}=\left(M_{l}^{2}+K_{l s}^{2}\right)^{1 / 2}, \\
K_{l c}=\int_{0}^{\infty} K(z) \cos l \Omega z d z, & K_{l s}=\int_{0}^{\infty} K(z) \sin l \Omega z d z .
\end{array}
$$

The formula (2.9) defines a stationary-wave type nonlinear correction term, which in general case of fading memory, describes two second harmonics, having double frequency in comparison with the basic harmonic (2.5) of the stationary part of the solution. One of the second harmonics propagates with the same phase velocity $c_{1}$ as the basic harmonic (2.5) and has the double damping decrement in comparison with the basic harmonic, while the other second harmonic the amplitude of which has the opposite sign, propagates with a different phase velocity $c_{2}$ and has another damping decrement $\gamma_{2}$.

If the strong inequalities

$$
K_{l s} \ll 1, \quad K_{l c} \ll 1
$$

are satisfied, which always takes place at great values of $\Omega$, then

$$
a_{l}=\frac{1}{2} K_{l s}-\frac{1}{4} K_{l s} K_{l c}+\ldots, \quad b_{l}=1+\frac{1}{2} K_{l c}+\frac{1}{8}\left(K_{l s}^{2}-K_{l c}^{2}\right)
$$

and the phase velocities $c_{1}$ and $c_{2}$ satisfying the condition $c_{1}<c_{2}<c$ do not differ essentially from $c$. In the limit case as $\Omega \rightarrow \infty$ we have $c_{1} \rightarrow c$ and $c_{2} \rightarrow c$.

It should be mentioned that an asymptotic solution for small values of $X$ describing the wave propagation in the nonlinear elastic medium is given in the form [ $\left.{ }^{4}\right]$

$$
U_{e}(X, t)=\varepsilon c\left[H\left(t_{1}\right)-H\left(t_{1}-t_{0}\right)\right] F_{e}(X, t),
$$

where

$$
F_{e}(X, t)=-\sin \Omega t_{1}-\frac{1}{4} \varepsilon k_{1} \Omega X c^{-1} \sin 2 \Omega t_{1}+\ldots .
$$

As will be shown in the next Section, the stationary solution given by (2.5) and (2.9) may be expanded into a series consisting of two parts $F_{0}^{*}(X, t)=F_{e}(X, t)+F_{v}(X, t)$. One of them, given by $(2.21)$, corresponds exactly to the solution of the nonlinear elastic problem while the other one describes the viscous properties of the medium.

Example 1. Let us consider a special case of standard viscoelastic body [11]. Then we have

$$
\begin{gathered}
K(t)=m \exp (-t / \tau) / \tau, \quad m=\text { const }>0, \\
K_{l s}=m l \Omega \tau / \omega l, \quad K_{l c}=m / \omega l, \quad \omega_{l}=1+l^{2} \Omega^{2} \tau^{2} .
\end{gathered}
$$

At great values of $\Omega$ the conditions (2.18) are satisfied and it follows from (2.10) and (2.19) that $\gamma_{1} \approx 2 \gamma_{2}$. The limit of $\gamma_{l}$ as $\Omega \rightarrow \infty$ is $\gamma_{l}=$ $=m /(l \tau c)$, i. e. at high frequencies the second harmonic of the second type is damped to the same degree as the basic harmonic. At small values of frequency we find $\gamma_{2} \approx 2 \gamma_{1}$ and for $\Omega \rightarrow 0$ we get

$$
\lim _{\Omega \rightarrow 0} \gamma_{l}=0, \quad \lim _{\Omega \rightarrow 0} c_{l}=c / \sqrt{1+m} .
$$


Consequently, at high frequencies the first-type second-order harmonic is damped quicker than the secondtype second-order harmonic. At low frequencies it is vice versa.

In Fig. 1 and $2 \gamma_{l}$ and $c_{l} / c$ are displayed against the frequency. The solid line 1 corresponds to the basic harmonic; the dashed lines 2 and 3 - to the second harmonics; the line 2 corresponds to the first type second harmonic and the line 3 corresponds to the second type second harmonic; $\tau=$ $=10^{-6} \mathrm{~s}, m=1, c=1200 \mathrm{~m} / \mathrm{s}$, $\varepsilon=0.01, k_{1}=3.5$.

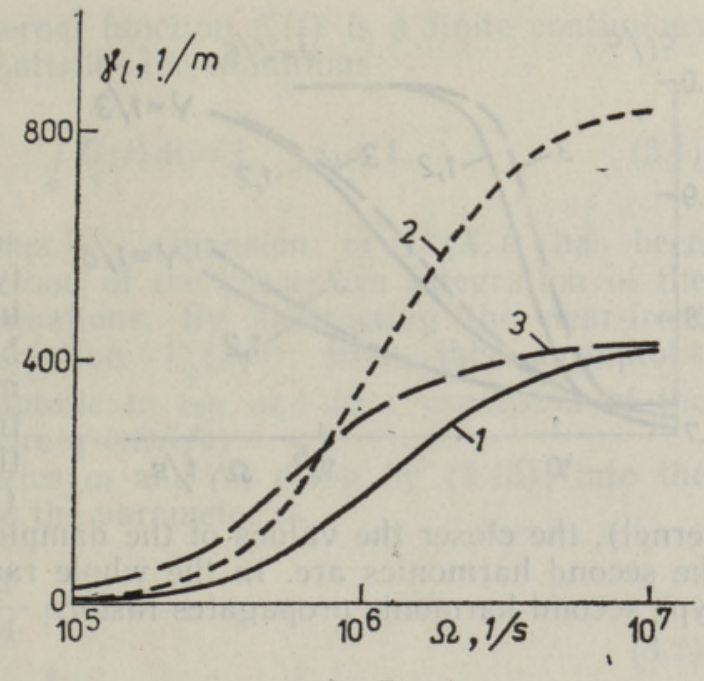

Fig. 1.

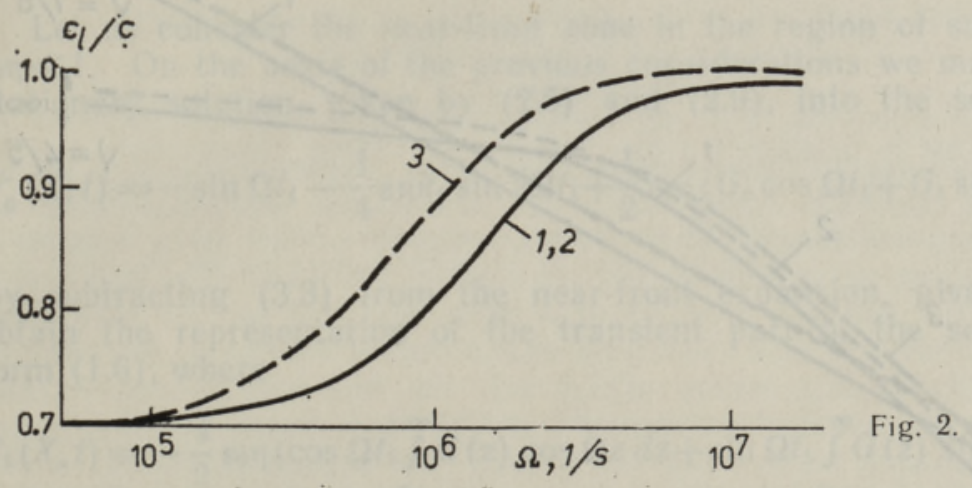

Example 2. Let us now consider the model of the hereditary elastic material with the Rabotnov function as an example of a singular kernel $\left[{ }^{12}\right]$

$$
\begin{aligned}
& K(t)=x t^{v-1} \sum_{n=0}^{\infty}(-\beta)^{n} t^{n v} / \Gamma[(n+1) v], \\
& 0<v<1, \quad 0<x<\infty, \quad 0<\beta<\infty,
\end{aligned}
$$

where $\Gamma(x)$ denotes the gamma-function. In case of this model we have the following expressions of $K_{l s}$ and $K_{l c}$

$$
\begin{array}{ll}
K_{l s}=x Q_{l} /\left(P_{l}^{2}+Q_{l}^{2}\right), & K_{l c}=x P_{l} /\left(P_{l}^{2}+Q_{l}^{2}\right), \\
P_{l}=(l \Omega)^{v} \cos (\pi v / 2)+\beta, & Q_{l}=(l \Omega)^{v} \sin (\pi v / 2) .
\end{array}
$$

It follows from the high-frequency approximation of $\gamma_{l}$

$$
\gamma_{l}(\Omega) \approx 2 \varkappa \sin (\pi v / 2) \Omega^{1-v} /\left(2 c l^{v}\right)
$$

that $\gamma_{1} \approx 2^{v} \gamma_{2}$ and $\lim _{\Omega \rightarrow \infty} \gamma_{l}=\infty$.

Following expressions describe the behaviour of $\gamma_{l}$ and $c_{l}$ at low frequencies

$$
\gamma_{2} \approx 2^{v} \gamma_{1}, \quad \lim _{\Omega \rightarrow 0} c_{l}=\frac{1}{\sqrt{2}} c, \quad \lim _{\Omega \rightarrow 0} \gamma_{l}=0,
$$




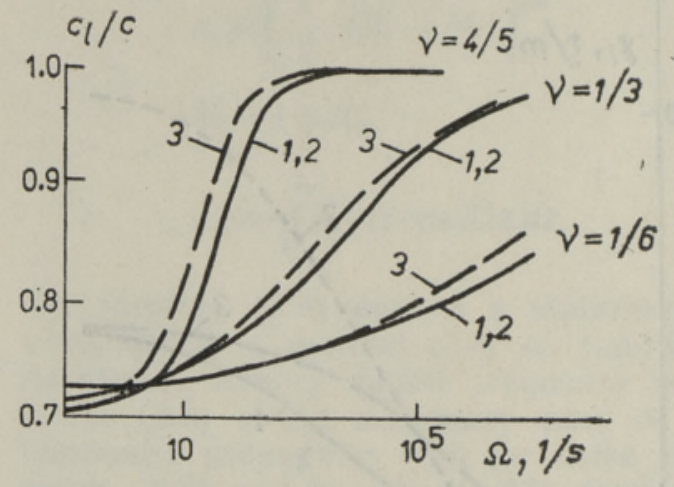

Fig. 3.

Consequently; far from the source $(a \Omega X / c \gg 1)$, the firsttype second harmonic dominates in the low-frequency region, but the second-type second harmonic dominates in the highfrequency region. The smaller the parameter of singularity $v$ (i. e. «the more singular» the kernel), the closer the values of the damping decrements of both types of the second harmonics are. In the whole range of frequencies the secondtype second harmonic propagates faster.

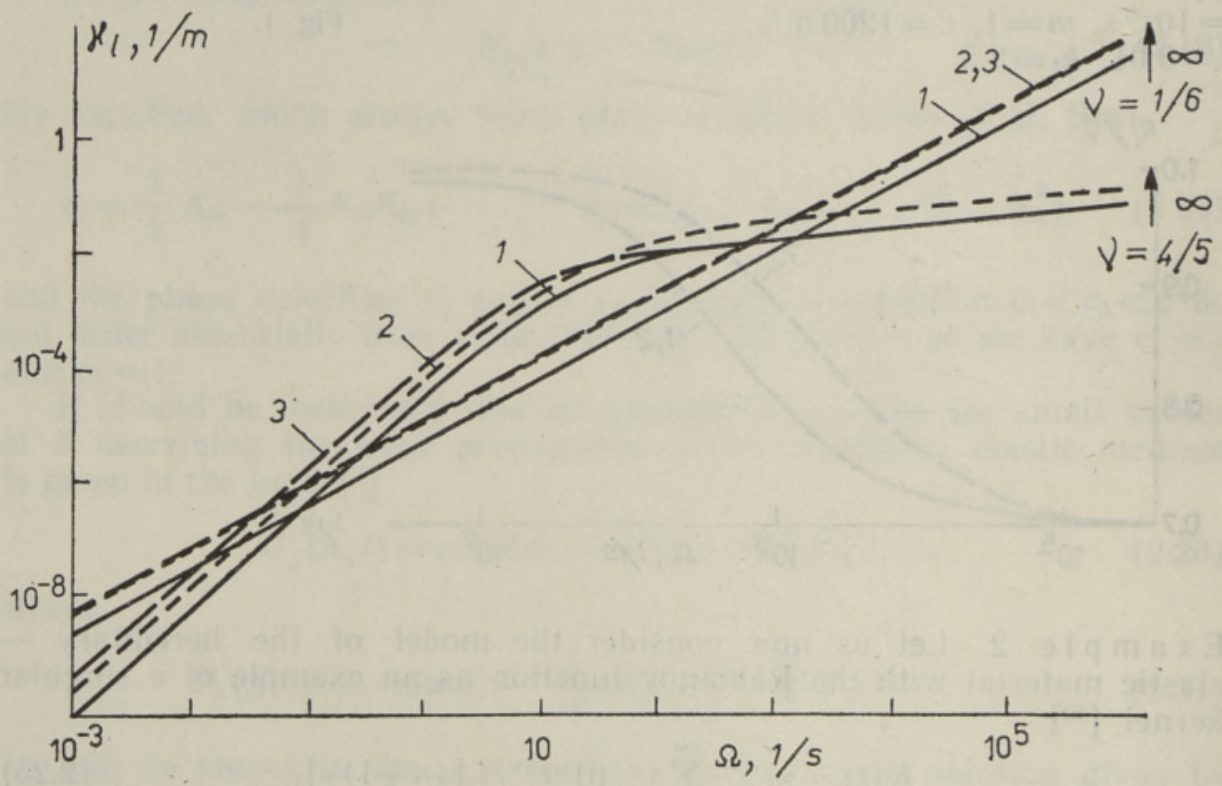

Fig. 4.

In Fig. 3 and 4 the behaviour of the damping decrements $\gamma_{1}, \gamma_{2}$ is shown, as well as the phase velocities $c_{1}, c_{2}$ against the frequency $\Omega$, respectively. In these Figs the solid line 1 corresponds to the basic harmonic, the dashed line 2 corresponds to the second harmonic of the first type, and the dotted line 3 corresponds to the second harmonic of the second type in the special case of $\beta=10 \mathrm{~s}^{-v}, x=10 \mathrm{~s}^{-v}, k_{1}=3.5$, $\varepsilon=0.1, c=1200 \mathrm{~m} / \mathrm{s}$ (laminated glass fabric).

\section{The transient part of the solution}

In this Section we shall show that the transient part of solution decays quickly behind the front and therefore its analytical representation may be obtained by making use of the asymptotic solution for the nearfront region, 
We shall assume that the kernel function $K(t)$ is a finite continuous and decreasing function which satisfies the conditions

$$
K(t)=\varepsilon_{0} G(t), \quad \int_{0}^{\infty} G(t) \mathrm{dt}=1, \quad \varepsilon_{0} \ll 1 .
$$

In $\left.{ }^{4}\right]$ the near-front asymptotic expansion of $U \cdot(X, t)$ has been derived with the aid of the method of the successive integration of the linear inhomogeneous wave equations. By subtracting the near-front expansion of the stationary solution $F_{0}^{*}(X, t)$ from this asymptotic expansion we obtain the asymptotic in $\varepsilon_{0} \eta$ and in $\varepsilon$ expansion of the transient part of the solution (here $\eta=\Omega X / c$ ).

We may expand the quantities $a_{l}$ and $b_{l}$, given by (2.15), into the asymptotic series with respect to the parameter $\varepsilon_{0}$

$$
\begin{aligned}
& a_{l}=\frac{1}{2} \varepsilon_{0} G_{l s}-\frac{1}{4} \varepsilon_{0}^{2} G_{l s} G_{l c}+\ldots, \\
& b_{l}=1+\frac{1}{2} \varepsilon_{0} G_{l c}+\frac{1}{8} \varepsilon_{0}^{2}\left(G_{l s}-G_{l c}\right)^{2}+\ldots .
\end{aligned}
$$

Let us consider the near-front zone in the region of small $X$, where $\varepsilon_{0} \eta \ll 1$. On the basis of the previous considerations we may expand the stationary solution, given by (2.5) and (2.9), into the series

$F_{0}^{*}(X ; t)=-\sin \Omega t_{1}-\frac{1}{4} \varepsilon \eta k_{1} \sin 2 \Omega t_{1}+\frac{1}{2} \varepsilon_{0} \eta\left(G_{c} \cos \Omega t_{1}+G_{s} \sin \Omega t_{1}\right)+\ldots$

By subtracting (3.3) from the near-front expansion, given in $\left[{ }^{4}\right]$, we obtain the representation of the transient part of the solution in the form (1.6), where

$F_{1}(X, t)=-\frac{1}{2} \varepsilon_{0} \eta\left(\cos \Omega t_{1} \int_{t_{1}}^{\infty} G(z) \cos \Omega z d z+\sin \Omega t_{1} \int_{t_{1}}^{\infty} G(z) \sin \Omega z d z\right)+\ldots$,

Fig. 5 .

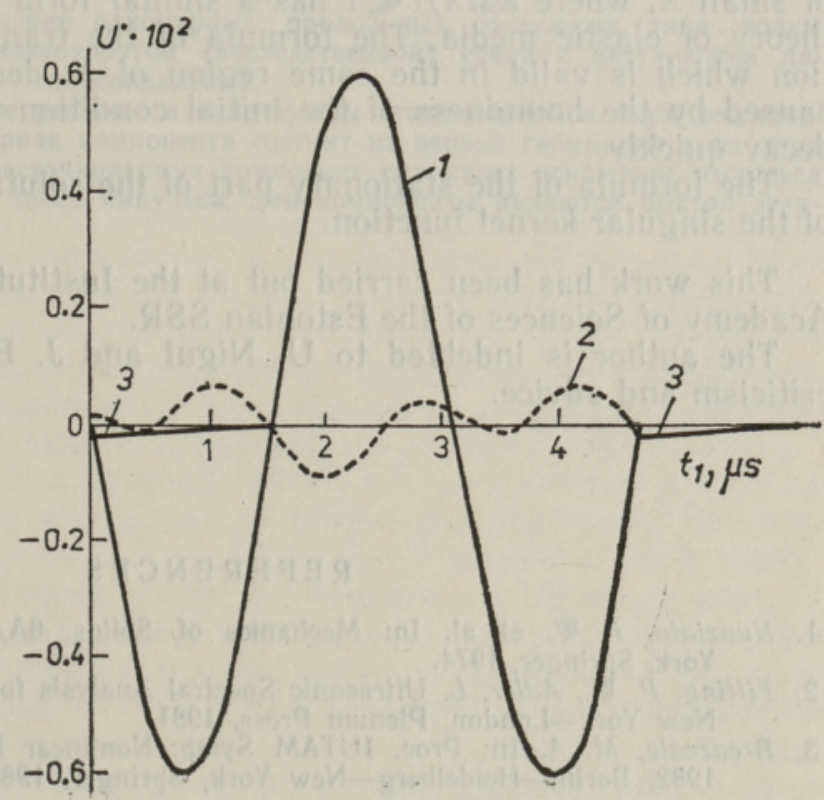


$F_{2}(X, t)=\frac{1}{2} \varepsilon_{0} \eta\left(\cos \Omega t_{1} \int_{t_{1}-t_{0}}^{\infty} G(z) \cos \Omega z d z+\sin \Omega t_{1} \int_{t_{1}-t_{0}}^{\infty} G(z) \sin \Omega z d z\right)+\ldots$

One of the transient components, $F_{1}(X, t)$ describes the distortion of the pulse behind the front and the other, $F_{2}(X, t)$ describes the tail of the pulse. Both components decay quickly.

$\mathrm{Example} 3$. In case of the standard viscoelastic body considered in Example 1, we have the kernel function (2.22) with $m=\varepsilon_{0}$ and, consequently, the transient part of the solution then reads

$$
\begin{gathered}
U_{N}(X, t)=-\frac{1}{2} \varepsilon \varepsilon_{0} c H\left(t_{1}\right) \eta \exp \left(-t_{1} / \tau\right) / \omega_{1}+ \\
+\frac{1}{2} \varepsilon \varepsilon_{0} c H\left(t_{1}-t_{0}\right) \eta \exp \left[\left(t_{0}-t_{1}\right) / \tau\right](-1)^{n} / \omega_{1}+\ldots .
\end{gathered}
$$

In Fig. 5 the velocity $U$ is plotted against the time $t_{1}$ at the fixed value of $X=1 \mathrm{~cm}$. Curve 1 - the basic harmonic, curve 2 - the sum of the second harmonics of the first and second type, curve 3 - the transient part; $\varepsilon=0.01, \varepsilon_{0}=0.1 ; c=6000 \mathrm{~m} / \mathrm{s}, \Omega=2 \cdot 10^{6} \mathrm{~s}^{-1}, \tau=10^{-6} \mathrm{~s}$.

\section{Conclusions}

In this paper an asymptotic solution is constructed consisting of a stationary and two transient components which describe the propagation of the one-dimensional longitudinal sine wave-type pulses in a nonlinear hereditary medium according to the regular kernel (3.1). The stationary component of the solution consists of a basic and two second harmonics which have different phase velocities and different damping decrements.

Far from the source, where $a \Omega X / c \gg 1$, the first-type second harmonic, which has the same phase velocity as the basic harmonic, dominates in the low frequency region, but the second-type second harmonic, which has a different phase velocity, dominates in the high-frequency region.

The asymptotic expansion of the stationary component for the region of small $X$, where $\varepsilon_{0} \Omega X / c \ll 1$ has a similar form to that known from the theory of elastic media. The formula of the transient part of the solution which is valid in the same region of $X$, describes two components caused by the boundness of the initial condition (1.5). Both components decay quickly.

The formula of the stationary part of the solution is also valid in case of the singular kernel function.

This work has been carried out at the Institute of Cybernetics of the Academy of Sciences of the Estonian SSR.

The author is indebted to U. Nigul and J. Engelbrecht for helpful criticism and advice.

\section{REFERENCES}

1. Nunziato, J. W. et al. In: Mechanics of Solids, 6A/4. Berlin-Heidelberg-New York, Springer, 1974. 2. Fitting, P.W., Adler, L. Ultrasonic Spectral Analysis for Nondestructive Evaluation,
New York-London. Plenum Press, 1981.

3. Breazeale, M. A. In: Proc. IUTAM Symp. Nonlinear Deformation Waves, Tallinn, 1982, Berlin-Heidelberg-New York, Springer, 1983, 332-348. 
4. Нигул У. К. Нелинейная акусто-диагностика. Л., «Судостроение», 1981.

5. Nigul, U. K. In: Proc. IUTAM Symp. Nonlinear Deformation Waves, Tallinn, 1982. Berlin-Heidelberg-New York, Springer, 1983, 225-272.

6. Engelbrecht, J. Wave Motion, № 1, 65-74 (1979).

7. Jeffrey, A., Engelbrecht, J. In: Wave Propagation in Viscoelastic Media. Research Notes in Mathematics, № 52. Boston-London-Melbourne, Pitman, 1982, 90116.

8. Rabotnov. Yu. N., Suvorova, Yu. V., Osokin, A. Ye. In: Proc. IUTAM Symp. Nonlinear Deformation Waves, Tallinn, 1982. Berlin-Heidelberg-New York, Springer, 1983, 157-170.

9. Иикович М., Нигул У. Изв. АН СССР. Мех. тверд. тела, № 1, $72-77$ (1984),

10. Карнаухов В. Т. Прикл. механика, 8, № 9, 91-96 (1972).

11. Cristensen, R. M. Theory of Viscoelasticity. New York etc., Acad. Press, 1982.

12. Работнов Ю. Н. Элементы наследственной механики твердых тел. М., «Наука», 1977.

\section{Research and Design Institute of Silicate Concrete}

Received

March 27, 1985

\section{Margarita ITSKOVITS \\ SINUSOIDAALSETE IMPULSSIDE LEVIK KUSTUVA MÄLUGA MITTELINEAARSES KESKKONNAS}

On vaadeldud ühemõõtmeliste deformatsioonilaine tüüpi pikiimpulsside levikut mittelineaarses viskoelastses kustuva mäluga keskkonnas. Eeldatakse, et algimpulss on sinusoid. Konstrueeritakse asümptootiline lahend statsionaarse ja kahe mittestatsionaarse komponendi summana. Statsionaarne komponent koosneb esimesest ja kahest teisest harmoonikust. Uks mittestatsionaarne komponent kirjeldab häiritust, mis tekib frondi taga, teine aga impulsi «sabạ»; mõlemad kustuvad kiiresti.

\section{Маргарита НЦКОВИЧ \\ РАСПРОСТРАНЕНИЕ СИНУСОИДАЛЬНЫХ ИМПУЛЬСОВ В НЕЛИНЕЙНОИ СРЕДЕ С ЗАТУХАЮЩЕИ ПАМЯТЬЮ}

Рассматривается распространение одномерных продольных импульсов типа волны деформации в нелинейной вязкоупругой (наследственной) среде с затухающей памятью. Начальный импульс - синусоидальный.

Строится асимптотическое решение в виде суммы стационарной и двух нестационарных компонент. Стационарная компонента состоит из первой гармоники и из двух вторых гармоник. Одна из нестационарных компонент описывает искажение импульса за фронтом, другая - т. н. хвост импульса. Эти компоненты являются быстро затухающими. 